INPLASY

PROTOCOL

To cite: Wang et al. The

Effectiveness of acupuncture

for metabolic syndrome: a

systematic review and meta-

analysis. Inplasy protocol

202210062. doi:

10.37766/inplasy2022.1.0062

Received: 12 January 2022

Published: 12 January 2022

Corresponding author:

Wang Xiaolan

1445750364@qq.com

Author Affiliation:

Changchun University of

Chinese Medicine

Support: $\mathbf{8 1 8 0 4 1 7 2 .}$

Review Stage at time of this submission: Preliminary

searches.

Conflicts of interest:

None declared.

\section{The Effectiveness of acupuncture for metabolic syndrome: a systematic review and meta-analysis}

Wang, XL1; Ha, LJ2; Yan, B33; Liu, XN4.

Review question / Objective: To evaluate the efficacy of different acupuncture in the treatment of metabolic syndrome.

Condition being studied: Metabolic syndrome (MS) is a state in which multiple diseases are clustered in the body, including hypertension, hyperglycemia, hyperlipidemia, and hyperuricemia, and its incidence is gradually increasing. The prevention and treatment of MS is a major international issue, and since MS and its complications are now internationally recognized as important risk factors for cardiovascular disease, the prevalence of metabolic syndrome has increased to the point of being considered an epidemic worldwide, especially in the last decade. In the United States, the prevalence of metabolic syndrome is estimated at $34 \%$ of the adult population. In addition, the growth rate of metabolic syndrome in developing countries is alarming. One can speculate that metabolic syndrome will have a huge impact on our lives. Acupuncture, as an external Chinese medical treatment, has been widely used in the treatment of metabolic syndrome. Electric acupuncture, buried thread, warm acupuncture, and ear acupuncture are all forms of acupuncture that play a role by stimulating acupoints. They are effective in the treatment of metabolic syndrome, easy to operate, and have no side effects. Therefore, this study used a meta-analysis to compare the efficacy of acupuncture on metabolic syndrome in order to provide some guidance for clinical treatment.

INPLASY registration number: This protocol was registered with the International Platform of Registered Systematic Review and Meta-Analysis Protocols (INPLASY) on 12 January 2022 and was last updated on 12 January 2022 (registration number INPLASY202210062).

\section{INTRODUCTION}

Review question / Objective: To evaluate the efficacy of different acupuncture in the treatment of metabolic syndrome.
Condition being studied: Metabolic syndrome (MS) is a state in which multiple diseases are clustered in the body, including hypertension, hyperglycemia, hyperlipidemia, and hyperuricemia, and its incidence is gradually increasing. The 
prevention and treatment of MS is a major international issue, and since MS and its complications are now internationally recognized as important risk factors for cardiovascular disease, the prevalence of metabolic syndrome has increased to the point of being considered an epidemic worldwide, especially in the last decade. In the United States, the prevalence of metabolic syndrome is estimated at $34 \%$ of the adult population. In addition, the growth rate of metabolic syndrome in developing countries is alarming. One can speculate that metabolic syndrome will have a huge impact on our lives. Acupuncture, as an external Chinese medical treatment, has been widely used in the treatment of metabolic syndrome. Electric acupuncture, buried thread, warm acupuncture, and ear acupuncture are all forms of acupuncture that play a role by stimulating acupoints. They are effective in the treatment of metabolic syndrome, easy to operate, and have no side effects. Therefore, this study used a meta-analysis to compare the efficacy of acupuncture on metabolic syndrome in order to provide some guidance for clinical treatment.

\section{METHODS}

Participant or population: Patients diagnosed with metabolic syndrome and have clear diagnostic criteria.

Intervention: The types of acupuncture in the intervention group will include: manual acupuncture, electric acupuncture, scalp acupuncture, earacupuncture, moxibustion, and fire needling.

Comparator: "Control measures will include: placebo/sham acupuncture or other interventions (such as drugs, physical therapies). ".

Study designs to be included: Randomized controlled trials (RCTs) will be included. Randomised controlled trials (RCTs) comparing acupuncture for Metabolic syndrome (MS) with no treatment, placebo, or conventional drugs (e.g. laxative agents) will be included. All eligible trials will be included regardless of language and publication types. Articles of the following research types will be excluded: case series, observational studies (including cohort and case-control studies) and retrospective studies, qualitative studies, animal experiments, review articles.

Eligibility criteria: Randomized clinical trials will be included irrespective of blinding, publication status or language.

Information sources: We will search articles in eight database including:CNKI,Wanfang, VIP, CBM, PubMed, Embase, Cochrane Library and web of science. All the publications, with no time restrictions, will be searched without any restriction of countries or article type. Reference list of all selected articles will independently screened to identify additional studies left out in the initial sea.

Main outcome(s): Results of changes in waist circumference, weight, blood pressure, blood glucose, blood lipid, and uric acid before and after treatment.

Quality assessment / Risk of bias analysis: The Cochrane systematic review of the risk of bias evaluation tool and Revman 5.3 software were used to evaluate the quality of the included literature. The six items of the quality evaluation were selection bias, performance bias, detection bias, attrition bias, reporting bias and other bias.

Strategy of data synthesis: ADDIS1.16.6 software was used for data analysis. ADDIS is a software that uses Bayesian framework and Markov Chain Monte Carlo (MCMC) to perform a priori evaluation and processing of data. Use the node cutting method provided by ADDIS software to evaluate whether direct evidence is consistent with indirect evidence, If $P>0.05$, select a consistency model for data analysis. Use potential scale reduction factor (PSRF) for convergence assessment, If the PSRF value is closer to 1 , the better the convergence effect is. Calculate the ranking probability of intervention measures based on the Bayesian network model to determine the intervention 
measures with the best curative effect. The outcome indicators in this paper are continuous variables, which are expressed by standard mean difference (SMD), and $95 \%$ confidence interval( $95 \% \mathrm{CI})$, respectively. Using Stata14.2 software to draw the network diagram.

Subgroup analysis: No subgroup analysis was performed.

Sensitivity analysis: Using the node cutting method provided by ADDIS software to evaluate whether direct evidence is consistent with indirect evidence, If $P>0.05$, select a consistency model for data analysis.

Language: No restriction on language.

Country(ies) involved: China.

Keywords: acupuncture therapy, network meta analysis, metabolic syndrome, body mass index, waist circumference, blood pressure, blood glucose.

Contributions of each author:

Author 1 - Wang Xiaolan.

Email: 1445750364@qq.com

Author 2 - Ha Lijuan.

Email: halij_1987@126.com

Author 3 - Yan Bing.

Email: yanbingdoctor@126.com

Author 4 - Liu Xiaona.

Email: 460398946@qq.com 